\title{
Evaporation, lifetime and robustness studies of liquid marbles for collision-based computing
}

\author{
Claire Fullarton $^{1}$, Thomas C. Draper ${ }^{1}$, Neil Phillips ${ }^{1}$, Richard Mayne ${ }^{1}$, \\ Ben P. J. de Lacy Costello ${ }^{2}$, and Andrew Adamatzky*1 \\ ${ }^{1}$ Unconventional Computing Laboratory, University of the West of England, Coldharbour Lane, Bristol, \\ UK \\ ${ }^{2}$ Institute of Biosensing Technology, Centre for Research in Biosciences, University of the West of \\ England, Coldharbour Lane, Bristol, UK
}

January 26, 2018

\begin{abstract}
Liquid marbles (LMs) have recently attracted interest for use as cargo carriers in digital microfluidics and have successfully been implemented as signal carriers in collisionbased unconventional computing circuits. Both application domains require LMs to roll over substantial distances and to survive a certain number of collisions without degrading. To evaluate the lifetime of LMs being subjected to movement and impact stresses, we have selected four types of coating to investigate: polytetrafluoroethylene (PTFE), ultra-high density polyethylene (PE), Ni, and a mixture of Ni with PE (Ni-PE). Hierarchies of robustness have been constructed which showed that pure PE LMs survived the longest when stationary and in motion. Pure PTFE LMs were shown to be the least resilient to multiple impacts and provided minimal protection against evaporative losses at small volumes $(2 \mu \mathrm{l}$ and $5 \mu \mathrm{l})$, but showed a good evaporative stability when stationary for larger LMs $(10 \mu \mathrm{l})$. Conversely, PE LMs showed a remarkable ability to withstand multiple impacts and were also stable when considering just passive evaporation. Hybrid Ni-PE LMs exhibited more resilience to multiple impacts compared to Ni LMs. Thus, when designing liquid marble devices it is paramount to determine impact pathways and select appropriate coating materials.
\end{abstract}

Keywords: Unconventional Computing, Liquid Marbles, Microfluidics, Particle-coated Droplets, Impact Dynamics, Logic Gates

\section{Introduction}

Liquid marbles (LMs), first reported by Aussillous and Quéré in 2001, [1] enabled a way of moving a liquid droplet across a surface without wetting it. These droplets were initially produced by encapsulating a liquid in a hydrophobic powder. The lack of contact the liquid inside the LM has with the surface avoids surface contamination problems and allows fast displacement of the LMs with minimal force. Aussillous and Quéré [1] found when investigating the motion of the LMs on an inclined plane, larger LMs moved slower than smaller ones, as predicted by Mahadevan and Pomeau. [2]

Compared to microchannel fluidic devices, LMs can move liquids without being confined to specific channels, meaning the droplets have the ability to move in many directions. This allows for more versatile fluidic devices and avoids any issues associated with channel wall adsorption found in traditional devices. As well as allowing the manoeuvrability of liquid, LMs have the

*Corresponding author. Email: Andrew.Adamatzky@uwe.ac.uk 
added benefit of being able to transport a powder to a particular place if required; once in the correct position the liquid can be evaporated. Exploiting the evaporation process has also been used to produce hollow spheres from LMs. [3, 4, 5]

Depending on the LMs carrier and cargo, LMs can be manipulated using electric and magnetic fields, which makes microfluidic applications of these particles possible. To ensure the LMs work efficiently in these microfluidic devices and as signal carriers in unconventional computing circuits, [6] it is important that the LMs are robust. [7]

The interactions between LMs vary depending on their coating and the liquid encapsulated within them. When LMs are pressed together or collide, they deform during impact but do not necessarily coalesce. [8] However, it has been observed that if the LMs are charged with a high enough voltage it is possible for the LMs to coalesce and subsequently merge. [9] Recently, it has been shown that LMs can coalesce depending on the force of the collision between the LMs and the type of LM coating. [6] It is possible to split a LM (ca. $1 \mathrm{~mm}$ diameter) into two smaller LMs due to this robustness, but it cannot be repeated as this would reduce the amount of hydrophobic coating on each of the resulting LMs, which means the LMs could then wet the surface. [10]

LMs can be produced by either rolling liquid droplets on a solid surface covered with a layer of hydrophobic powder, e.g. lycopodium, extracted from moss spores, or by mixing a hydrophobic powder in water. It is possible to also encapsulate liquids using hydrophilic particles (e.g. carbon black). [11] Normally, the powder coatings are treated to make sure the LMs are robust (e.g. treating lycopodium with fluoro-decyl-trichlorosilane or treating silica with fluorosilane). The powder shells of the LMs are permeable to gas which allows them to be used for gas sensing applications. For example, $\mathrm{NH}_{3}$ detection has successfully been demonstrated with polytetrafluoroethylene (PTFE) coated LMs. [12]

To tailor the properties of LMs for a variety of chemical, biological and engineering focused applications, a wide range of coating and liquid combinations have been investigated and extensively reviewed in the literature. [13, 14, 15, 16, 17, 18] Typically, aqueous solutions and glycerol have been used as the liquid for the LMs, with glycerol being used to vary the viscosity of the encapsulated liquid. Using high surface tension liquids (e.g. polar liquids) ensures the hydrophobic coating is effective, which is why Aussillous and Quéré used a mixture of water and glycerol. Recently, non volatile solvents e.g. ionic liquids, have been used as a way of reducing evaporation of the liquid from LMs.

Changing the size of the particles used for the coating can vary the properties of LMs. Bhosale et al. [19] highlighted that LMs made from microparticulates are opaque, which means it is not possible to optically monitor the liquid inside. Therefore, they used nanoparticulates which made the LMs transparent, allowing for the LMs to be optically monitored during transport. This is beneficial for the use in biofluidic devices e.g. if cells are being transported or stored in the LMs, it is therefore possible to monitor any changes in the encapsulated biological fluid. Bhosale et al. [20] produced water and glycerol LMs coated with fumed silica which had been treated with hexamethyldisilazane (nHMDS) and dimethyldichlorosilane (mDMDCS). Glycerol, due to its low volatility was used to prepare LMs for imaging using an Environmental Scanning Electron Microscope.

Magnetic fields do not have a significant effect on LMs prepared from water and coated with only lycopodium grains. However, the hydrophobic coating and / or the encapsulated liquid can be tailored to enable interaction with a magnetic field. [21] LMs coated with hydrophobic iron powder mixed with lycopodium were attracted to a magnet. It was also reported possible to add iron powder into the encapsulated liquid, which initially made the LM attract to the magnet however, the iron powder from inside the LM attached to the magnet and the clean LM dropped back down on to the surface. The LMs produced by both these methods had issues with robustness, with either the iron particles detaching from the surface or particles aggregating, resulting in droplet deformation in the magnetic field. To overcome these issues, 
Zhao et al. [22] have produced water filled LMs with $\mathrm{Fe}_{3} \mathrm{O}_{4}$ nanoparticles. The robust LMs produced were able to be reversibly opened and closed using a magnetic field. Both these properties are beneficial for the use of these objects in microfludic devices and micro-reactors. Zhao et al. [22] demonstrated the facile movement of the LMs in a magnetic field, the ease of opening / closing the LMs, adding and removing liquid from the open LMs, coalescence of the LMs within the magnetic field as well as using the $\mathrm{Fe}_{3} \mathrm{O}_{4}$ nanoparticles to remove a water droplet from a hydrophilic surface.

It is crucial to understand how the LMs behave under different conditions, with various coatings and encapsulated liquids, to gauge their suitability for use in microfluidic applications and for the purposes of our research, a LM interaction gate for collision-based computing. [6] A collision-based, or dynamical, computation employs mobile compact finite patterns, mobile self-localisations, in active non-linear medium or travelling mechanical devices. [23] There are several sources of collision-based computing. The ideas of colliding signals, existing from the nineteenth century in physics and physiology, have been put in an automata framework, when papers by Atrubin, on multiplication in cellular automata [24], Fisher, on generation of prime numbers in cellular automata [25], and Waksman, on eight-state solution for a firing squad synchronisation problem [26] were published. Berlekamp, Conway and Guy proved that the Game of Life 2D cellular automaton (with just two cell states and eight cell neighbourhood) can imitate computers. [27] Gliders, small patterns of non-resting cell states, were selected as the main carriers of information. The localisations travel in space and do computation when they collide with each other. Essentials of collision-based computing are as follows. Information values (e.g. truth values of logical variables) are given by either absence or presence of the localisations or other parameters of the localisations. The localisations travel in space and do computation when they collide with each other. There are no predetermined stationary wires: a trajectory of the travelling pattern is a transient wire. The localisations undergo transformations, e.g. change velocities, form bound states, annihilate or fuse when they interact with other mobile patterns. Information values of localisations are transformed as a result of collision and thus a computation is implemented. In our case, collision-based computation is performed by observing the change (or lack there of) in the vector of, for example, LMs. Two LMs are propelled towards each other, if the LMs continue unhindered then there has been no collision, and if the pathway changes then there has been a collision. Using this logic gates can be constructed, such as AND, AND-NOT, NOR. [28]

Therefore, it is important to focus on: the lifetime of LMs in terms of how they evaporate (dependant on their coating and liquid cargo); the robustness and lifetime when rolling and in collisions with both other LMs and different surfaces; how the preparation of LMs affects the coating quality; and how different coatings and liquid cargo affect the properties of the LMs.

An overview of existing published results show that the liquid and the coating can have a varying effect on the lifetime and evaporation rates of LMs. $[29,16]$ It has been reported that LMs can have either slower, faster or similar evaporation rates compared to bare water droplets. [30]

Dandan and Erbil [31] studied the evaporation periods and lifetimes of graphite LMs (prepared using volumes of $5 \mu \mathrm{l}$ and $10 \mu \mathrm{l}$ ) and found that the LMs had nearly double the lifetime in comparison to bare water droplets. The results were compared to the behaviour of LMs produced by Bhosale et al., [19] who prepared water and glycerol LMs coated with PTFE, nHMDS, mDMDCS micropowders at $25^{\circ} \mathrm{C}$ with $20 \% \mathrm{RH}$.

Tosun and Erbil [32] studied the evaporation of PTFE LMs (between $21^{\circ} \mathrm{C}$ to $26^{\circ} \mathrm{C}$ ). The PTFE (medium particle size $5 \mu \mathrm{m}-9 \mu \mathrm{m}$ ) used to prepare the LMs was found to aggregate (cluster size $20 \mu \mathrm{m}-50 \mu \mathrm{m}$ ) and form multilayers (up to $100 \mu \mathrm{m}$ ). LMs $(5 \mu \mathrm{l})$ were reported to collapse after 26 min. The PTFE LMs had longer evaporation times compared to bare water droplets. The LMs were found to retain their shape for $20-50 \%$ of the evaporation period. The PTFE coating on the LM was reported to reduce the evaporation by $25-45 \%$ compared to water depending 
on the relative humidity (RH). LMs were reported to have a lifetime of $26 \mathrm{~min}(54 \% \mathrm{RH})$ and $60 \min (66 \% \mathrm{RH})$.

Aberle et al. investigated the evaporation of LMs when heated to high temperatures and found above a certain temperature LMs behave similar to Leidenfrost droplets. [33] When LMs evaporate at room temperature, the process is controlled by mass transfer i.e. driven by the vapour concentration gradient.

Impact dynamics of LMs has very recently been investigated by Supakar et al. [34] LMs were prepared from water and coated with hydrophobised glass beads. Crack formation and spreading of the LMs occurred upon impact. The particles on the underside of the LM prevented contact with the solid substrate until the spacing between them reached a critical value $(300 \mu \mathrm{m}-$ $800 \mu \mathrm{m})$, then the LM attached to the surface. [34]

In this work, we analyse the properties of LMs with four types of coating: polytetrafluoroethylene (PTFE), polyethylene (PE), Ni and a hybrid coating consisting of a mixture of Ni and PE (Ni-PE). We selected these coatings because PTFE and PE are well accepted models of LM coatings, they are inert materials, with good mechanical and wear properties, and exhibit high resistances to impacts. Ni particles show magnetic properties and therefore are ideal candidates for devices where non-contact retaining of particles with magnets is required. [35, 36, 6, 37, 38] A mixture of $\mathrm{Ni}$ and $\mathrm{PE}$ was chosen because this coating has proven to be practical in the implementation of collision-based computing devices with LMs. [6]

\section{Experimental Section}

LMs were prepared using deionised water as the encapsulated liquid (volumes $2.5 \mu \mathrm{l}, 5 \mu \mathrm{l}$ and $10 \mu \mathrm{l}$ ) and coated with either polytetrafluoroethylene (Alfa Aesar, $(6 \mu \mathrm{m}-10 \mu \mathrm{m})$, CAS 900284-0, Product Code 44184), ultra high density polyethylene (Sigma Aldrich, CAS 9002-884, Product Code 1002018483), Ni (GoodFellow Metals, 99.8\%, $(4 \mu \mathrm{m}-7 \mu \mathrm{m})$, Product Code NI006021/1) and a mixture of Ni and PE (Ni-PE). For all evaporation studies, the mass as a function of time was recorded using an Adventurer Ohaus balance fitted with a closed glass case over the balance. The glass case was sealed with parafilm to maintain a controlled environment with which to monitor the mass changes of the LMs and bare water droplets. Temperature and humidity was monitored using a TH165 Prime Capsule Data Logger (temperature measurement accuracy $\pm 0.3^{\circ} \mathrm{C}$ at $25^{\circ} \mathrm{C}$, display resolution $0.1^{\circ} \mathrm{C}$; humidity measurement accuracy $\pm 2 \% \mathrm{RH}$ at $\left.25^{\circ} \mathrm{C}\right)$. Stationary LM evaporation rates were recorded for $5 \mathrm{LMs}$ for each volume $(2.5 \mu \mathrm{l}, 5 \mu \mathrm{l}$ and $10 \mu \mathrm{l}$ ) per coating. LMs were prepared by pipetting the required volume from a distance of ca. $5 \mathrm{~mm}$ from the top of a powder bed. The water droplet was rolled on the powder bed for $10 \mathrm{~s}$ to produce a LM. The prepared LM was transferred to a fresh weighing boat and rolled for $5 \mathrm{~s}$ to remove any non-adhered powder coating, prior to recording the initial mass of the LM. Masses of LMs were recorded until dryness to determine stationary evaporation rates (Tab. 1(c,d)).

For rolling and lifetime testing, LMs $(10 \mu \mathrm{l})$ were prepared using the method described above. The LMs were transferred to a fresh weighing boat after formation. This weighing boat was placed into another attached to the top of an orbital shaker. The LMs were confined whilst rolling to the area of the weighing boat. Experiments were conducted to monitor the evaporation rate and the lifetime of rolling LMs. Evaporation rates were recorded for 5 LMs per coating. The masses of PTFE, PE and Ni-PE LMs were recorded every 5 minutes after a period of rolling (parameters are $10 \mathrm{~s}$ stationary, $4 \mathrm{~min} 30 \mathrm{~s}$ rolling at $140 \mathrm{rpm}, 20 \mathrm{~s}$ stationary). Only one Ni LM survived this rolling regime (lasting $15 \mathrm{~min}$ ) giving an evaporation rate of $0.29 \mathrm{mg} \mathrm{min}^{-1}$, with most Ni LMs only lasting 5-10 min. Therefore, different parameters (10 s stationary, $30 \mathrm{~s}$ rolling at $140 \mathrm{rpm}, 20 \mathrm{~s}$ stationary) were used to record the rolling evaporation rate for Ni LMs shown in Tab. 2(a,b).

LMs $(10 \mu \mathrm{l})$ of each of the four coatings were subjected to impact testing onto a polystyrene surface (Petri dish). LMs were placed onto the end of a modified plastic pipette fashioned 
into a spoon shape, suspended parallel to the top surface of a stack of Petri dishes. The lid of the top Petri dish was rotated between LM drops to create a fresh impact surface. LMs were dropped from varying heights $(1-4 \mathrm{~cm})$ depending on how well the LMs survived the impact. For each drop height, the impacts of 10 LMs were observed. Videos of the LMs were recorded at $1000 \mathrm{fps}$ and a resolution of 1280x720, with an fps1000HD-256 (Imagetec Ltd). Video playback was conducted at $25 \mathrm{fps}$, giving an effective slowdown of 40x. Videos can be viewed in the Supporting Information.

Impact tests were also conducted in a continuous manner, using a custom-constructed rolling drop test device, similar in design to an alternating ladder. The ladder steps (Fig. 1) were constructed of $3 \mathrm{~mm}$ acrylic, which were then attached (RS Pro AB-3 Liquid Acrylic Adhesive) to another solid sheet of $3 \mathrm{~mm}$ acrylic. The back of the device was coated in graphite (Kontakt-Chemie Graphit 33), in order to reduce the build up of static charge. All videos were filmed in the same manner as previously described and an example video is available in the Supporting Information.

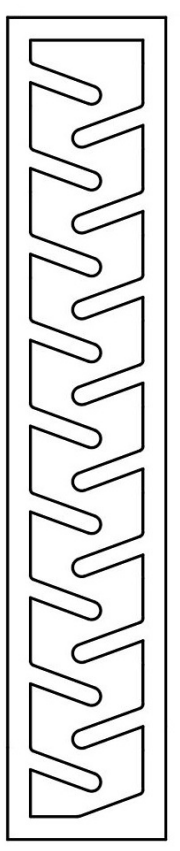

(a)

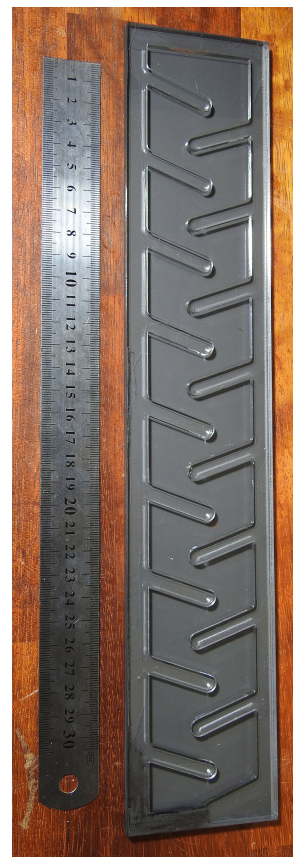

(b)

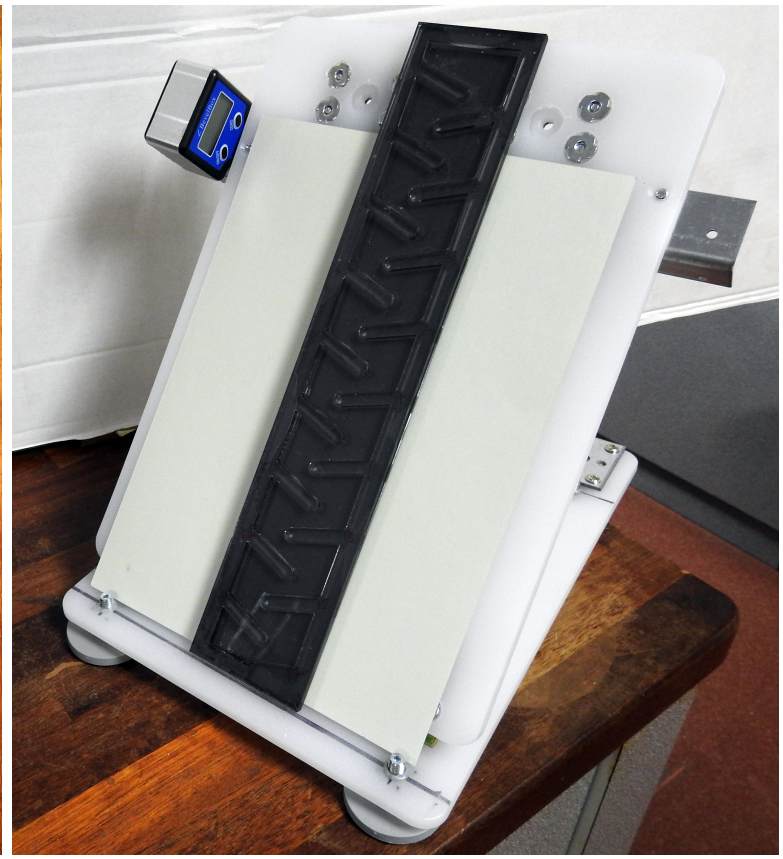

(c)

Figure 1: The (a) design schematic and $(b, c)$ photographs of the rolling drop test device. A LM rolls from top to bottom. Individual steps of the ladder are $25 \mathrm{~mm}$ in length with a drop of $13 \mathrm{~mm}$.

For all measurements the device was angled at $52^{\circ}$ from horizontal. The ladder was mounted on a bespoke slanted platform with adjustable pitch from $30^{\circ}$ to $90^{\circ}$. The pitch angle was manually adjusted by means of M10-1.5 screw thread. The pitch angle was measured with a digital gauge, full range $+/-180^{\circ}$ with $+/-0.1^{\circ}$ resolution. The gauge was zeroed using 1800 $\mathrm{mm}$ Beam Level with accuracy of $0.029^{\circ}$ in standard position. The base of the platform has four adjustable legs which are set to level the rig. To conduct a test, a single LM (10 $\mu$ l) was placed at the top of the device and allowed to roll $25 \mathrm{~mm}$ at $20^{\circ}$ before a drop of $13 \mathrm{~mm}$. This rolling-dropping process was then repeated down the full run of the ladder (17 steps). If the LM was still intact after rolling down the full ladder, it was re-positioned at the top for another run. The number of steps the LM survived before structural failure indicated the robustness of the LM after multiple impacts.

Low-power light microscope images were produced using an MX4T stereomicroscope (Brunel, 
Table 1: Parameters of LMs and their evaporation rates. (a) Particle size $(\mu \mathrm{m})$ (b) Average mass (mg) of coating for different volume ( $\mu \mathrm{l}$ ) LMs (c) Evaporation rates $\left(\mathrm{mg} \mathrm{min}{ }^{-1}\right.$ ) of stationary LMs with different initial liquid volumes ( $\mu \mathrm{l}$ ) (5 LMs for each volume $(2.5 \mu \mathrm{l}, 5 \mu \mathrm{l}$ and $10 \mu \mathrm{l})$ per coating studied) (d) Evaporation rates $\left(\mathrm{mg} \mathrm{s}^{-1}\right)$ as a function of LM surface area $\left(\mathrm{m}^{2}\right)$ (e) Evaporation rates $\left(\mathrm{mg} \mathrm{min}{ }^{-1}\right)$ of bare water droplets ( $\mathrm{f}$ ) Average time (min) to dryness for stationary LMs

(a)

\begin{tabular}{cccc}
\hline PTFE & PE & Ni & Ni-PE \\
\hline $6-10$ & 100 & $4-7$ & $4-7$ and 100 \\
\hline
\end{tabular}

(b)

\begin{tabular}{ccccc}
\hline Volume $(\mu \mathrm{l})$ & PTFE & PE & Ni & Ni-PE \\
\hline 2.5 & $<0.1$ & 0.8 & 0.68 & 0.64 \\
5 & $<0.1$ & 1.0 & 1.06 & 0.9 \\
10 & $<0.1$ & 1.78 & 1.7 & 1.66 \\
\hline
\end{tabular}

(c)

\begin{tabular}{ccccc}
\hline Volume $(\mu \mathrm{l})$ & PTFE & PE & Ni & Ni-PE \\
\hline 2.5 & $0.0612 \pm 0.0074$ & $0.0545 \pm 0.0046$ & $0.0560 \pm 0.0099$ & $0.0458 \pm 0.0022$ \\
5 & $0.0818 \pm 0.0102$ & $0.0636 \pm 0.0019$ & $0.0640 \pm 0.0057$ & $0.0648 \pm 0.0054$ \\
10 & $0.0962 \pm 0.0109$ & $0.0911 \pm 0.0074$ & $0.1011 \pm 0.0082$ & $0.1052 \pm 0.0045$ \\
\hline
\end{tabular}

(d)

\begin{tabular}{ccccc}
\hline Volume $(\mu \mathrm{l})$ & PTFE & PE & Ni & Ni-PE \\
\hline 2.5 & $29.38 \pm 3.55$ & $35.80 \pm 3.02$ & $46.73 \pm 8.26$ & $30.90 \pm 1.48$ \\
5 & $25.08 \pm 3.13$ & $26.34 \pm 0.79$ & $25.57 \pm 2.28$ & $25.35 \pm 2.11$ \\
10 & $17.49 \pm 1.98$ & $20.85 \pm 1.69$ & $22.99 \pm 1.86$ & $24.68 \pm 1.06$ \\
\hline
\end{tabular}

(e)

\begin{tabular}{cc}
\hline Volume $(\mu \mathrm{l})$ & Water \\
\hline 2.5 & $0.0797 \pm 0.0047$ \\
5 & $0.0924 \pm 0.0096$ \\
10 & $0.1227 \pm 0.0057$ \\
\hline
\end{tabular}

(f)

\begin{tabular}{cccccc}
\hline Volume $(\mu \mathrm{l})$ & PTFE & PE & Ni & Ni-PE & Water \\
\hline 2.5 & $38.6 \pm 5.4$ & $46.5 \pm 5.5$ & $36.5 \pm 7.3$ & $54.2 \pm 5.4$ & $30.3 \pm 4.1$ \\
5 & $59.8 \pm 9.6$ & $74.3 \pm 3.7$ & $75.2 \pm 4.9$ & $76.2 \pm 6.3$ & $53.9 \pm 5.6$ \\
10 & $104.9 \pm 13.5$ & $110.3 \pm 10.5$ & $101.6 \pm 10$ & $97.9 \pm 9.3$ & $82.1 \pm 4.6$ \\
\hline
\end{tabular}

UK) and a 16MP smart phone camera (G953F, Samsung, South Korea), via an adapter. All four varieties of LM were prepared using $5 \mu \mathrm{l}$ of deionised water containing $0.25 \%$ carboxylatemodified fluorescent latex microspheres (Sigma, Germany), $2 \mu \mathrm{l}$ in diameter. Fluorescence imaging was performed using Axiovert 200M inverted microscope (Zeiss, Germany) and a coherent $488 \mathrm{~nm}$ LED light source. Images were captured with an SC50 camera (Olympus, Japan). No image post-processing was performed. 
Table 2: Parameters of rolling LMs and their evaporation rates. (a) Evaporation rates ( $\mathrm{mg} \mathrm{min}^{-1}$ ) of rolling LMs with an initial liquid volume of $10 \mu$ l (Rolling regime parameters for PTFE, PE and Ni-PE LMs - $10 \mathrm{~s}$ stationary, $4 \mathrm{~min} 30 \mathrm{~s}$ rolling at $140 \mathrm{rpm}, 20 \mathrm{~s}$ stationary, rolling regime parameters for Ni LMs - $10 \mathrm{~s}$ stationary, $30 \mathrm{~s}$ rolling at $140 \mathrm{rpm}, 20 \mathrm{~s}$ stationary) (5 LMs per coating studied) (b) Evaporation rates ( $\mathrm{mg} \mathrm{s}^{-1}$ ) of rolling LMs reported in (a) as a function of LM surface area $\left(\mathrm{m}^{2}\right)$ (c) Average time (min) LMs rolled for, in the rolling evaporation experiments

(a)

\begin{tabular}{cccc}
\hline PTFE & PE & Ni & Ni-PE \\
\hline $0.2216 \pm 0.0172$ & $0.1724 \pm 0.0139$ & $0.1604 \pm 0.0139$ & $0.21028 \pm 0.0185$ \\
\hline
\end{tabular}

(b)

\begin{tabular}{cccc}
\hline PTFE & PE & Ni & Ni-PE \\
\hline $82.71 \pm 6.42$ & $88.97 \pm 7.17$ & $457.54 \pm 39.65$ & $127.45 \pm 11.21$ \\
\hline
\end{tabular}

(c)

\begin{tabular}{cccc}
\hline PTFE & PE & Ni & Ni-PE \\
\hline $27.9 \pm 5.3$ & $37.8 \pm 6.1$ & $2.5 \pm 0.4$ & $27.1 \pm 0.2$ \\
\hline
\end{tabular}

Table 3: Calculated parameters of LMs (reported in (Tab. 1(c)) subjected to evaporation studies. (a) Surface area $\left(\mathrm{mm}^{2}\right)$ of LMs (b) Radius ( $\mathrm{mm}$ ) of non-deformed LMs (c) Approximate values for the Young's modulus (Pa) of LMs

(a)

\begin{tabular}{ccccc}
\hline Volume $(\mu \mathrm{l})$ & PTFE & PE & Ni & Ni-PE \\
\hline 2.5 & 34.72 & 42.06 & 33.75 & 38.48 \\
5 & 54.35 & 59.62 & 61.08 & 63.24 \\
10 & 91.70 & 100.39 & 98.69 & 97.54 \\
\hline
\end{tabular}

(b)

\begin{tabular}{ccccc}
\hline Volume $(\mu \mathrm{l})$ & PTFE & PE & Ni & Ni-PE \\
\hline 2.5 & 1.66 & 1.83 & 1.64 & 1.75 \\
5 & 2.08 & 2.18 & 2.20 & 2.24 \\
10 & 2.70 & 2.83 & 2.80 & 2.79 \\
\hline
\end{tabular}

(c)

\begin{tabular}{ccccc}
\hline Volume $(\mu \mathrm{l})$ & PTFE & PE & Ni & Ni-PE \\
\hline 2.5 & 173 & 157 & 176 & 165 \\
5 & 138 & 132 & 131 & 128 \\
10 & 107 & 102 & 103 & 103 \\
\hline
\end{tabular}




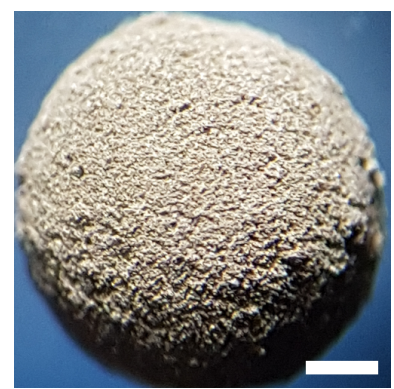

(a)

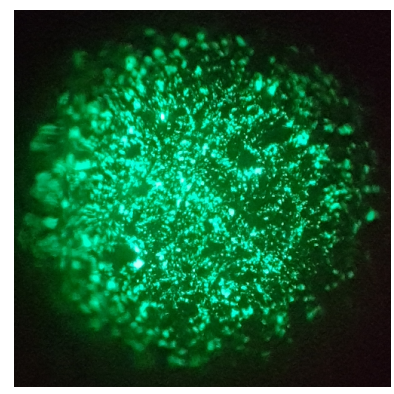

(e)

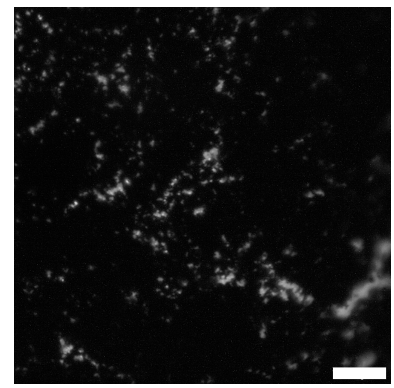

(i)

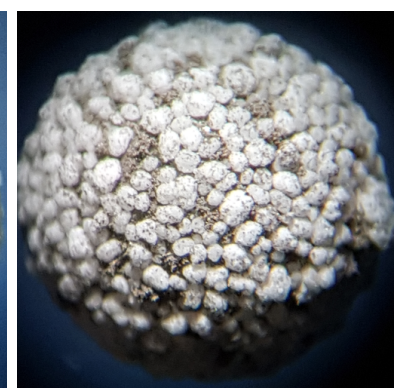

(b)

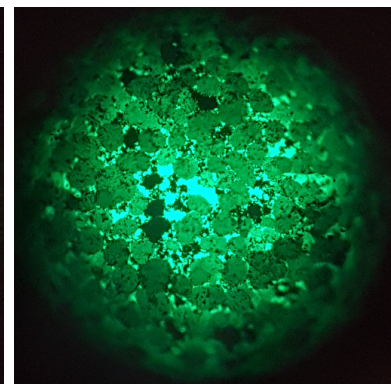

(f)

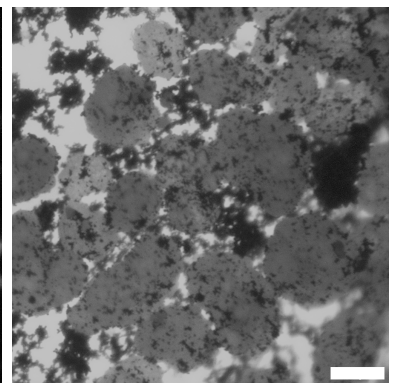

(j)

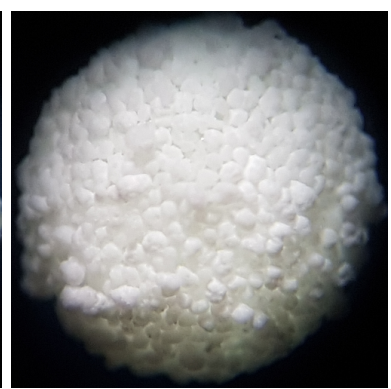

(c)

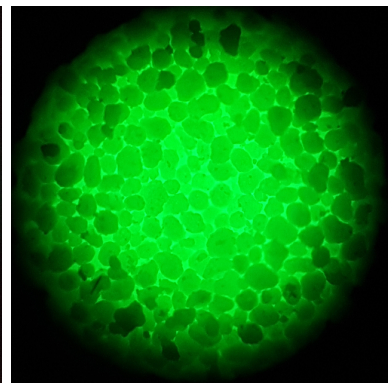

(g)

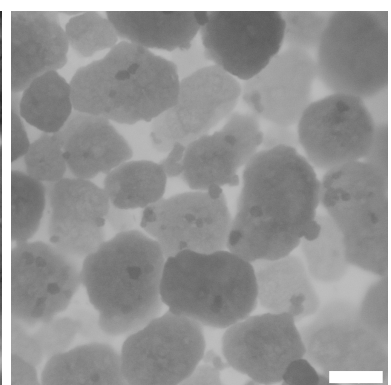

(k)

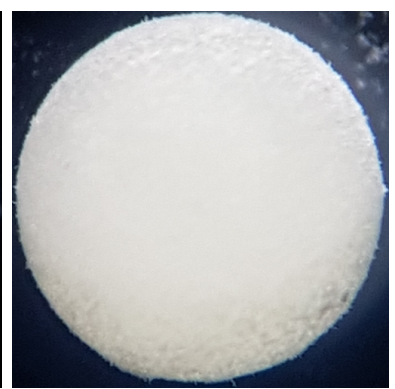

(d)

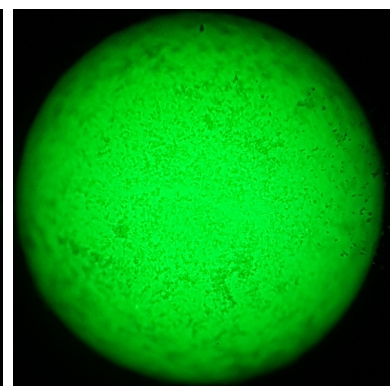

(h)

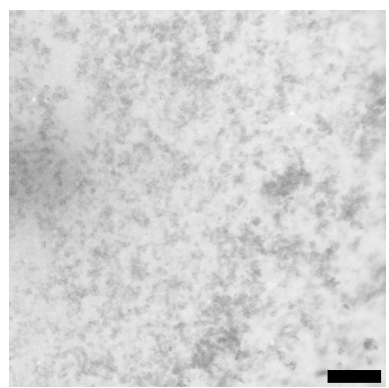

(1)

Figure 2: LM microscopy. (a-d) Stereoscopic images. (e-h) Fluorescence microscopy, low power. (i-l) Fluorescence microscopy, high power. Scale bars: [a-h] $500 \mu \mathrm{m}$, [i-l] $100 \mu \mathrm{m}$. LM coatings $(a, e, i)$ Nickel. $(b, f, j)$ Nickel-PE. $(c, g, k)$ PE. $(d, h, l)$ PTFE. 


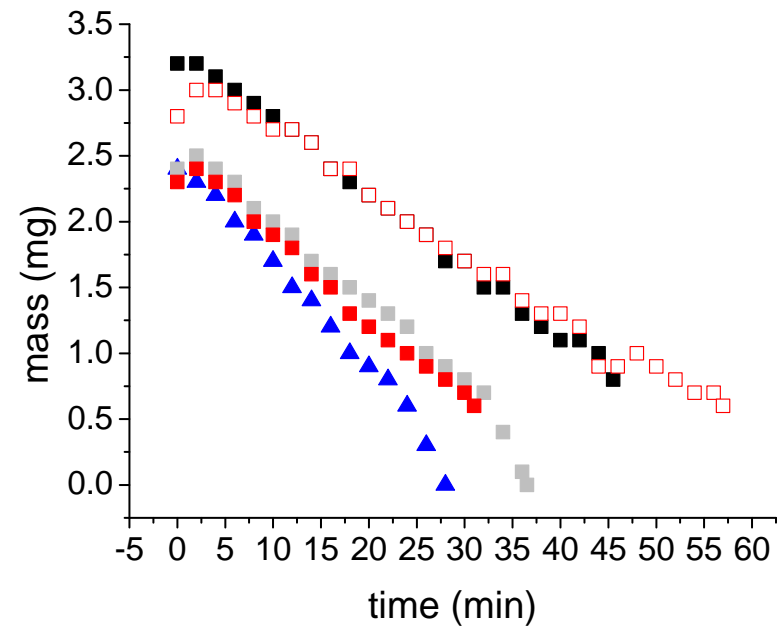

(a)

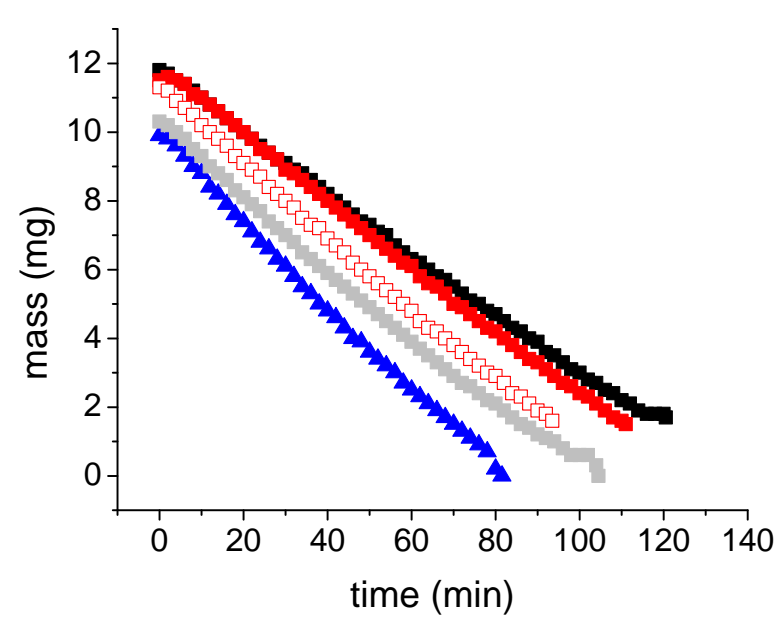

(c)

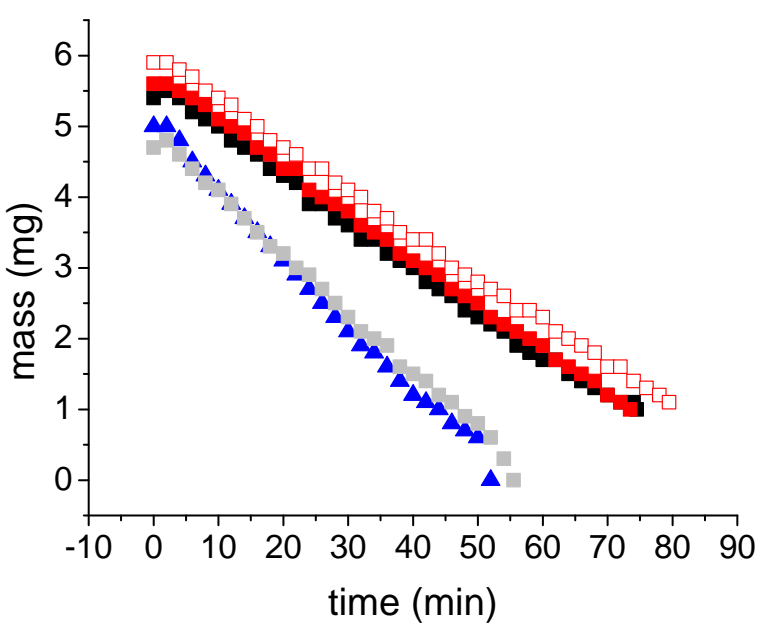

(b)

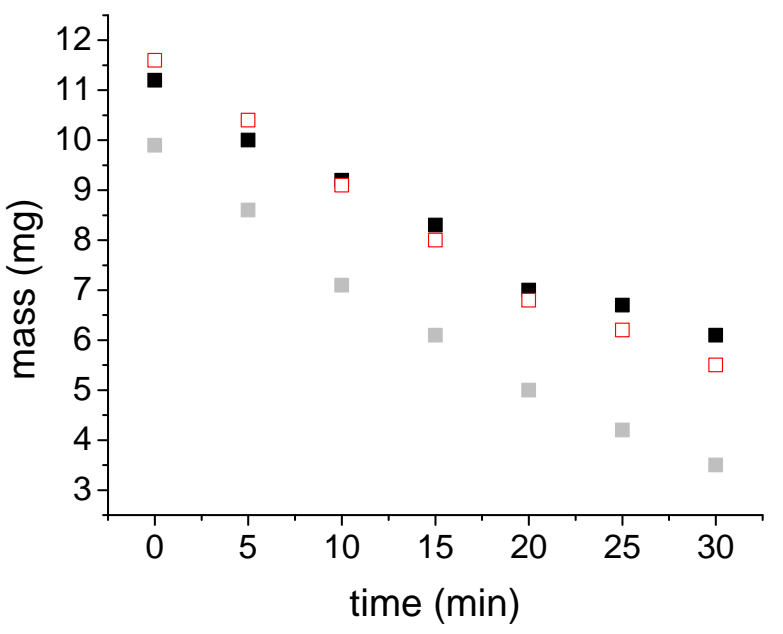

(d)

Figure 3: Representative mass transfer plots of (a) $2.5 \mu$ l stationary, (b) $5 \mu$ l stationary, (c) $10 \mu \mathrm{l}$ stationary, (d) $10 \mu \mathrm{l}$ rolling LMs coated with PE (black squares), Ni (red squares), Ni-PE (red outline squares), PTFE (light grey squares) compared to a bare water droplet (blue triangles) of the same volume. 


\section{Results and Discussion}

Initially, to understand the lifetime of various LMs, four different powder coatings were used to study the evaporation rates of stationary LMs under ambient conditions. These conditions were comparable with conditions reported for the evaporation of LMs in the literature. The masses of the powder coatings for PE, Ni and Ni-PE LMs increased with increasing volume (proportional to the LM volumes) (Tab. 1(b)). The masses of the coatings for PTFE LMs were less than $0.1 \mathrm{mg}$, but their masses may vary between volumes, however this was unable to be detected due to the accuracy of the balance. All LMs (volumes $2.5 \mu \mathrm{l}, 5 \mu \mathrm{l}$ and $10 \mu \mathrm{l}$ ) prepared from all four coatings exhibited slower stationary evaporation rates compared to a bare water droplet under the same conditions (Tab. 1(c,e) and Fig. 3(a-c)). This suggests that the LMs prepared had particle coatings consisting of multilayers rather than monolayers. If the coatings were monolayers of particles, the LMs would dry at a similar rate or even at a faster rate than bare water droplets, as observed by Laborie et al. for water LMs prepared using polystyrene particles. [30] The presence of multilayers for the four LM coatings investigated are confirmed from microscope images of the LMs (Fig. 2). LMs formed from powders with a smaller particle size (Tab. 1(a)), PTFE, sub $5 \mu \mathrm{m}$ and Ni, 5-10 $\mu \mathrm{m}$, tended to form fine layers around the internal fluid with high amounts of continuity in the layer (see Fig. 2(i-1)), whereas inter-particle gaps were comparatively large between the irregularly sized 100-200 $\mu \mathrm{m}$ PE particles, which were observed to exceed $75 \mu \mathrm{m}$ (Tab. 1(a)). In the case of the hybrid Ni-PE LMs, the characteristically large gaps in the powder layer were partially filled by the smaller Ni particles.

The surface areas $\sigma$ of the LMs were determined using Eq. (1); where $m$ is the recorded mass of the LMs in (mg) and $\rho$ is the encapsulated liquid density (in this case, water, $0.998 \mathrm{mg}$ $\left.\mathrm{cm}^{-3}\right)$. [20]

$$
\sigma=4 \pi\left(\frac{6 m}{\pi \rho}\right)^{2 / 3}
$$

Surface areas reported in Tab. 3(a) enabled the determination of evaporation rates (mg $\mathrm{s}^{-1}$ ) as a function of surface area $\left(\mathrm{m}^{2}\right)$; reported in Tab. 1(d) for stationary LMs and Tab. 2(b) for rolling LMs. The evaporation rate $\left(\mathrm{mg} \mathrm{s}^{-1}\right)$ as a function of surface area $\left(\mathrm{m}^{2}\right)$ provided a means of comparing the evaporation rates of different LM volumes. Stationary LMs, reported in Tab. 1(d), gave very similar values for all four coatings, slight increases were noted for $2.5 \mu \mathrm{l}$ LMs which were assumed to be attributed to the early onset of the coating buckling and increased stresses in the coating. Rolling LMs, reported in Tab. 2(b), gave very similar evaporation rates for PTFE and PE coating however LMs coated with Ni based coatings gave much greater evaporative losses whilst rolling.

Surface areas of LMs determined using Eq. (1) also enabled the determination of a rough approximation of the effective Young's modulus $E$ of the LMs Eq. (2), assuming a spherical geometry to determine the non-deformed radius $R_{0}$ of the LM and assuming the effective surface tension $\gamma_{\text {eff }}$ is equal to that of water $\left(72 \mathrm{mN} \mathrm{m}^{-1}\right)$. [39] The effective Young's modulus $E$ for each of the LMs (prepared for stationary evaporation studies using all four coatings) ranged between $102 \mathrm{~Pa}-176 \mathrm{~Pa}$. Values increased with increasing volume which suggested small volume LMs were less elastic than larger volume LMs.

$$
E \approx \frac{4 \gamma_{\mathrm{eff}}}{R_{0}}
$$

For mechanical applications of LMs, it is important to understand how the lifetime of LMs vary when moving across a surface, compared to studying just the stationary properties. For PE LMs $(10 \mu \mathrm{l})$, the evaporation increased upon rolling by $89 \%$ cf. stationary LMs. For PTFE LMs $(10 \mu \mathrm{l})$, the evaporation rate increased upon rolling by $130 \% \mathrm{cf}$. stationary LMs. For Ni-PE $(10 \mu \mathrm{l}$ ), the evaporation rate increased upon rolling by $100 \% \mathrm{cf}$. stationary LMs (Tab. 2(a) and Fig. 3(d)). For Ni LMs, as mentioned in the experimental section, only one Ni LM survived the 
rolling regime (parameters: $10 \mathrm{~s}$ stationary, $4 \mathrm{~min} 30 \mathrm{~s}$ rolling at $140 \mathrm{rpm}, 20 \mathrm{~s}$ stationary), lasting $15 \mathrm{~min}$, giving an evaporation rate of $0.29 \mathrm{mg} \mathrm{min}^{-1}$, an increase in evaporation rate of $187 \%$, however most of the Ni LMs only lasted 5-10 min during this rolling regime, which made determining an evaporation rate challenging. Therefore, different parameters were used to record the rolling evaporation rate shown in Tab. 2(a). Both the change in the rolling parameters needed for $\mathrm{Ni}$ LM rolling experiments and the minimal average rolling time (2.5 min) of these LMs reported in Tab. 2(c) shows that these LMs are not robust and have a short rolling lifetime, meaning this coating is not suitable for LM applications that require prolonged movement. The PE LMs had the longest lifetime when rolling.

In impact studies on fresh LMs, 100\% of PE LMs survived impact when dropped from $2 \mathrm{~cm}$, $60 \%$ survived when dropped from $2.5 \mathrm{~cm}, 0 \%$ survived when dropped from $3 \mathrm{~cm} .100 \%$ of $\mathrm{Ni}$ LMs survived impact when dropped from $2 \mathrm{~cm}, 80 \%$ survived when dropped from $2.5 \mathrm{~cm}, 30 \%$ survived when dropped from $3 \mathrm{~cm}, 0 \%$ survived when dropped from $4 \mathrm{~cm} .100 \%$ of Ni-PE LMs survived impact when dropped from $2 \mathrm{~cm}, 0 \%$ survived when dropped from $2.5 \mathrm{~cm} .100 \%$ of PTFE LMs survived a drop from $1 \mathrm{~cm}$, while only $10 \%$ survived a drop from $2 \mathrm{~cm}, 0 \%$ survived when dropped from $3 \mathrm{~cm}$. Slow motion videos for the LMs that survived impact (for all four coatings) show the spreading and subsequent bouncing of the LMs. LMs that did not survive were shown to form powder coated sessile drops upon impact. The individual impact tests determined PTFE LMs to be the weakest LMs when impacting onto a solid surface. Ni LMs were shown to survive the greatest drop, despite not performing well in rolling studies.

In addition to the stationary drop testing, the LMs were also tested for their ability to withstand multiple impacts by conducting a continuous rolling drop test. The LMs were rolled on a $20^{\circ}$ slope for $25 \mathrm{~mm}$, before dropping $13 \mathrm{~mm}$ onto the lower level and repeating the process. A device with 17 drops was constructed, and any LMs that survived one run of the ladder were re-positioned back at the top of the ladder for another run. Each LM type was tested 10 times, with the averages and standard deviations (SD) shown in Tab. 4.

Table 4: Average number of drops survived by a LM of a particular coating, using the continuous rolling drop test device and the accompanying standard deviation (SD)

\begin{tabular}{ccccc}
\hline & PTFE & PE & Ni & Ni-PE \\
\hline Average & 15.1 & 186.1 & 16.8 & 28.9 \\
SD & 2.3 & 137.1 & 6.4 & 15.5 \\
\hline
\end{tabular}

These results indicated that the pure $\mathrm{Ni}$ and pure PTFE LMs were the least resilient to multiple impacts. This is not surprising, as both were shown to perform relatively poorly in comparison to $\mathrm{Ni}$ and Ni-PE in evaporation and impact studies reported above. For the Ni LMs, this may be attributed to the relatively high wettability of the coating, with a small impact more likely to completely wet the $\mathrm{Ni}$, bringing the encapsulated liquid in contact with the substrate and consequently breaking the LM. For the PTFE LMs, this is a result of the thin layer of particles formed on the LM (in contrast to other coatings). [32] Such a thin coating presents a minimal barrier to surface wetting.

PE LMs showed a remarkable ability to withstand multiple impacts (Tab. 4), with the best performing LM surviving 547 drops on the ladder. This is likely due to a combination of the large contact angle of PE on the surface, and the slightly larger particle size. It should be noted that robustness of the PE coating varied significantly between LMs. The poorest performing PE LM survived 68 drops, which still outperformed the other LM coatings investigated. The large variation in robustness of PE LMs was attributed to problematic static charge build-up. This was partially offset by coating the back of the device with conductive graphite, to help dissipate the static charge build-up.

Hybrid Ni-PE LMs were observed to have an intermediate robustness cf. Ni LMs and PE LMs. Therefore, the robustness advantage gained for including PE in the hybrid LM coating were 
offset by the presence of Ni. From the microscope image in Fig. 2(b), it can be seen that there are some small areas of pure $\mathrm{Ni}$ on the hybrid LM. One can speculate that these areas are weak points in the coating and the cause of the decrease in robustness during impact cf. PE LMs.

For two types of LMs $A$ and $B$ we write $A>B$ if LM $A$ survives longer than LM $B$. The following hierarchies of survival (robustness) were discovered in experimental laboratory studies:

- Stationary 2.5 $\mu \mathrm{l}$ : LMs: Ni-PE $>\mathrm{PE} \approx \mathrm{Ni}>\mathrm{PTFE}$

- Stationary $5 \mu \mathrm{l}: \mathrm{PE} \approx \mathrm{Ni} \approx \mathrm{Ni}-\mathrm{PE} \gg \mathrm{PTFE}$

- Stationary $10 \mu \mathrm{l}: \mathrm{PE}>\mathrm{PTFE}>\mathrm{Ni}>\mathrm{Ni}-\mathrm{PE}$

- Rolling $10 \mu \mathrm{l}: \mathrm{PE}>\mathrm{Ni}-\mathrm{PE}>\mathrm{PTFE}>\mathrm{Ni}$

- Survival on impact hierarchy $10 \mu \mathrm{l}: \mathrm{Ni}>\mathrm{PE}>\mathrm{Ni}-\mathrm{PE}>\mathrm{PTFE}$

- Continuous dropping $10 \mu \mathrm{l}: \mathrm{PE}>\mathrm{Ni}-\mathrm{PE}>\mathrm{Ni}>\mathrm{PTFE}$

There are no strong trends between all the hierarchies of survival and hierarchies of LMs based on the particle sizes (Tab. 1(a)) and coating masses of the LMs (Tab. 1(b)). However, the survival hierarchy of rolling LMs matches that of the hierarchy of the particle sizes, i.e. LMs with larger size coating particles survive longer.

Recently, LMs have been shown to be of use in unconventional computing. [6] Such computations result from the collisions of LMs in interaction gates, and the resulting deflected pathways. Using this logic, it is possible to perform mechanical digital Boolean algebra. [28, 40] The ability to select and use a LM based on its properties is an important tool for computing with LMs. A computationally useful LM needs to be dynamically sturdy in order to survive the number of rebounds and collisions required of it; additionally it needs to be long lasting with regards to its evaporative decay. The longer lasting the LM is, the more complicated the calculations that can be performed with it; with the longest lasting LMs able to be recycled through the device again.

Current work with LM interaction gates [6] indicate that processing times for a half-adder would be approximately $300 \mathrm{~ms}$. This is inclusive of the magnetic synchronisation. A full-adder is composed of two half-adders, therefore we can extrapolate that a full $n$-bit adder would require approximately $600 \mathrm{~ms}$. Or, more universally, an $n$-bit adder would require $0.6 n$ seconds to process. Consequently, a typical 64-bit arithmetic calculation would take approximately $38.4 \mathrm{~s}$ per cycle of addition. It should be noted that these values are empirically derived maximum calculation times, and not theoretical minimum calculation times. There is much scope for the calculations to be performed quicker as this field develops.

When these calculation times are compared with our LM lifetimes and durability results, it can be seen that the PE LM is well suited for use in collision-based LM computing. This is due to its ability to withstand impacts, combined with its reduced evaporation rate. Alternatively, if magnetic properties are required then the system may need to be designed around the reduced impact robustness of the Ni-PE LM.

Implementation of a full one-bit adder requires at most two collisions between LMs. [6] From the experimental data on a number of drops a LM can survive (Tab. 4) we can speculate that a reliable 14-bit adder can be realised with Ni-PE coated LMs, while the largest possible adder with PE coated LMs would be over 250-bits.

\section{Conclusions}

The lifetime of LMs subjected to movement and impact stresses has been assessed by investigating stationary evaporation rates, evaporation rates of rolling LMs, impact testing and continuous rolling drop tests. Pure PTFE LMs were shown to be the least resilient to multiple impacts and provided minimal protection against evaporative losses at small volumes $(2 \mu \mathrm{l}$ and 
$5 \mu \mathrm{l})$, but showed a good evaporative stability when stationary for larger LMs $(10 \mu \mathrm{l})$. Pure Ni were shown to withstand the greatest drop in single impact tests, however did not perform well during multiple impact testing and evaporation studies. PE LMs were determined to be the best performing marbles in terms of both impact and evaporative stability. These type of studies are paramount in supporting the optimisation of LMs for both LM based microfluidic devices and collision-based computing. For the latter, it is important to balance optimal resilience with functionality, therefore PE-Ni hybrid LMs were produced which were magnetic (beneficial for controlled capture and release of the LMs) but have enhanced rolling and multiple impact resilience compared to pure Ni marbles. Hybrid systems allow many degrees of freedom in terms of tunability of desirable properties.

\section{Conflict of interest}

There are no conflicts to declare.

\section{Acknowledgements}

This research was supported by the EPSRC with grant EP/P016677/1 "Computing with Liquid Marbles".

\section{Supporting Information}

Electronic supporting information is available, showing slow motion videos of LM impact studies.

\section{References}

[1] Aussillous, P.; Quéré, D. Liquid marbles, Nature 2001, 411, 924-927.

[2] Mahadevan, L.; Pomeau, Y. Rolling droplets, Phys. Fluids 1999, 11, 2449-2453.

[3] Hapgood, K. P.; Khanmohammadi, B. Granulation of hydrophobic powders, Powder Technol. 2009, 189, 253 - 262.

[4] Eshtiaghi, N.; Arhatari, B.; Hapgood, K. P. Producing hollow granules from hydrophobic powders in high-shear mixer granulators, Adv. Powder Technol. 2009, 20, 558-566.

[5] Guan, Y.; Meng, X.; Qiu, D. Hollow Microsphere with Mesoporous Shell by Pickering Emulsion Polymerization as a Potential Colloidal Collector for Organic Contaminants in Water, Langmuir 2014, 30, 3681-3686.

[6] Draper, T. C.; Fullarton, C.; Phillips, N.; de Lacy Costello, B. P.; Adamatzky, A. Liquid marble interaction gate for collision-based computing, Mater. Today 2017, 20, 561-568.

[7] Aussillous, P.; Quéré, D. Properties of liquid marbles, Proc. R. Soc. A Math. Phys. Eng. Sci. 2006, 462, 973-999.

[8] Bormashenko, E.; Pogreb, R.; Balter, R.; Aharoni, H.; Bormashenko, Y.; Grynyov, R.; Mashkevych, L.; Aurbach, D.; Gendelman, O. Elastic properties of liquid marbles, Colloid Polym. Sci. 2015, 293, 2157-2164.

[9] Liu, Z.; Fu, X.; Binks, B. P.; Shum, H. C. Coalescence of electrically charged liquid marbles, Soft Matter 2016, 13, 119-124.

[10] Bormashenko, E.; Bormashenko, Y. Non-stick droplet surgery with a superhydrophobic scalpel, Langmuir 2011, 27, 3266-3270.

[11] Bormashenko, E.; Pogreb, R.; Musin, A. Stable water and glycerol marbles immersed in organic liquids: From liquid marbles to Pickering-like emulsions, J. Colloid Interface Sci. 2012, 366, 196-199. 
[12] Bormashenko, E. Liquid marbles: Properties and applications, Curr. Opin. Colloid Interface Sci. 2011, 16, 266-271.

[13] Bormashenko, E. Liquid marbles, elastic nonstick droplets: From minireactors to selfpropulsion, Langmuir 2017, 33, 663-669.

[14] Bormashenko, E. New insights into liquid marbles, Soft Matter 2012, 8, 11018-11021.

[15] Fujii, S.; Yusa, S. I.; Nakamura, Y. Stimuli-Responsive Liquid Marbles: Controlling Structure, Shape, Stability, and Motion, Adv. Funct. Mater. 2016, 26, 7206-7223.

[16] McHale, G.; Newton, M. I. Liquid marbles: topical context within soft matter and recent progress, Soft Matter 2015, 11, 2530-2546.

[17] Ooi, C. H.; Nguyen, N. T. Manipulation of liquid marbles, Microfluid. Nanofluidics 2015, 19, 483-495.

[18] Rycheckỳ, O.; Majerská, M.; Král, V.; Štěpánek, F.; Čejková, J. Spheroid cultivation of HT-29 carcinoma cell line in liquid marbles, Chemical Papers 2017, 1-9.

[19] Bhosale, P. S.; Panchagnula, M. V.; Stretz, H. A. Mechanically robust nanoparticle stabilized transparent liquid marbles, Appl. Phys. Lett. 2008, 93, 034109.

[20] Bhosale, P. S.; Panchagnula, M. V. On synthesizing solid polyelectrolyte microspheres from evaporating liquid marbles, Langmuir 2010, 26, 10745-10749.

[21] Nguyen, N. T. Deformation of ferrofluid marbles in the presence of a permanent magnet, Langmuir 2013, 29, 13982-13989.

[22] Zhao, Y.; Fang, J.; Wang, H.; Wang, X.; Lin, T. Magnetic liquid marbles: Manipulation of liquid droplets using highly hydrophobic $\mathrm{Fe}_{3} \mathrm{O}_{4}$ nanoparticles, Adv. Mater. 2010, 22, 707-710.

[23] Adamatzky, A., Ed.; Collision Based Computing; 2002.

[24] Atrubin, A. A one-dimensional real-time iterative multiplier, IEEE Transactions on Electronic Computers 1965, 394-399.

[25] Fischer, P. C. Generation of primes by a one-dimensional real-time iterative array, Journal of the ACM (JACM) 1965, 12, 388-394.

[26] Balzer, R. An 8-state minimal time solution to the firing squad synchronization problem, Information and Control 1967, 10, 22-42.

[27] Berlekamp, E. R.; Conway, J. H.; Guy, R. K. "Winning Ways for your Mathematical Plays, Vol. 2", 1982.

[28] Fredkin, E.; Toffoli, T. Conservative logic. In Collision-based computing; Springer: 2002.

[29] McHale, G.; Newton, M. I. Liquid marbles: principles and applications, Soft Matter 2011, 7, 5473-5481.

[30] Laborie, B.; Lachaussée, F.; Lorenceau, E.; Rouyer, F. How coatings with hydrophobic particles may change the drying of water droplets: incompressible surface versus porous media effects, Soft Matter 2013, 9, 4822-4830.

[31] Dandan, M.; Erbil, H. Y. Evaporation rate of graphite liquid marbles: Comparison with water droplets, Langmuir 2009, 25, 8362-8367. 
[32] Tosun, A.; Erbil, H. Y. Evaporation rate of PTFE liquid marbles, Appl. Surf. Sci. 2009, 256, 1278-1283.

[33] Aberle, C.; Lewis, M.; Yu, G.; Lei, N.; Xu, J. Liquid marbles as thermally robust droplets: coating-assisted Leidenfrost-like effect, Soft Matter 2011, 7, 11314-11318.

[34] Supakar, T.; Kumar, A.; Marston, J. O. Impact dynamics of particle-coated droplets, Phys. Rev. E 2017, 95, 1-10.

[35] Zhao, Y.; Xu, Z.; Parhizkar, M.; Fang, J.; Wang, X.; Lin, T. Magnetic liquid marbles, their manipulation and application in optical probing, Microfluid. Nanofluidics 2012, 13, 555-564.

[36] Zhao, Y.; Xu, Z.; Niu, H.; Wang, X.; Lin, T. Magnetic liquid marbles: Toward "Lab in a droplet", Adv. Funct. Mater. 2015, 25, 437-444.

[37] Nguyen, N.-T.; Hejazian, M.; Ooi, C. H.; Kashaninejad, N. Recent Advances and Future Perspectives on Microfluidic Liquid Handling, Micromachines 2017, 8, 186.

[38] Huang, G.; Li, M.; Yang, Q.; Li, Y.; Liu, H.; Yang, H.; Xu, F. Magnetically Actuated Droplet Manipulation and Its Potential Biomedical Applications, ACS Appl. Mater. Interfaces 2017, 9, 1155-1166.

[39] Whyman, G.; Bormashenko, E. Interpretation of elasticity of liquid marbles, J. Colloid Interface Sci. 2015, 457, 148-151.

[40] Toffoli, T. Reversible computing. In International Colloquium on Automata, Languages, and Programming; 1980. 


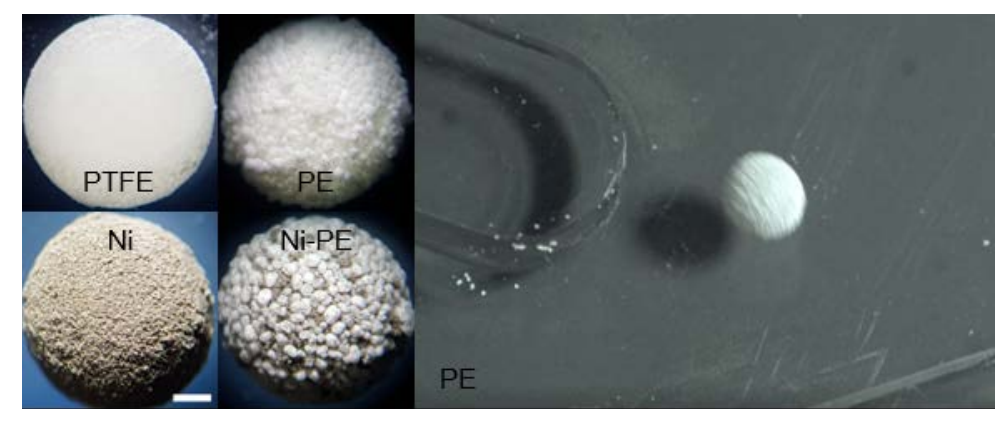

Figure 4: For Table of Contents only 